\title{
IMPORTÂNCIA DO GERENCIAMENTO DE REJEITOS RADIOATIVOS NA IODOTERAPIA
}

Maria Lúcia Rissato**
LucianaC. Oliveira***
Maria Lúcia Ribeiro***
Marcus César Avezum Alves de Castro****
Natália N. Ferreira****

\section{Resumo:}

A iodoterapia é uma das terapêuticas da medicina nuclear que emprega como radionuclídeo o iodo radioativo. Esse tratamento, realizado em unidades de internação, exige procedimentos de radioproteção para prevenir contaminação de pacientes, equipe de saúde e meio ambiente. Uma discussão sobre a aplicação dessa terapêutica mostra a importância da implantação de programas de gerenciamento de resíduos radioativos, gerados em unidades hospitalares.

Palavras-chave: Iodoterapia; Medicina Nuclear; Rejeitos Radioativos.

\section{Radioatividade: uso e proteção}

A humanidade vem convivendo com a radioatividade, através de fontes naturais de radiação e artificiais, tais como: uso de raios-x na medicina, chuvas de partículas radioativas produzidas pelos testes de armas nucleares, acidentes naturais com materiais radioativos, entre outros (Figura 1). A radioatividade natural é mais conhecida com átomos "pesados", normalmente com número atômico acima do chumbo $(\mathrm{Pb}: \mathrm{Z}=82)$. Entretanto, pode-se, atualmente, fabricar isótopos radioativos de todos os elementos, por meio de bombeamento de um núcleo estável. O urânio235 , o césio-137, o cobalto- 60 e o tório- 232 são exemplos de elementos fisicamente

*Mestre do Programa de Mestrado em Desenvolvimento Regional e Meio Ambiente- Centro Universitário de Araraquara - Uniara.

**Doutora em Química e Pesquisadora do Instituto de Química de Araraquara - Unesp Araraquara-SP.

***Professores doutores docentes do Programa de Mestrado em Desenvolvimento Regional e Meio Ambiente - Centro Universitário de Araraquara - Uniara.

*****Bolsista de Apoio Técnico do Programa de Mestrado em Desenvolvimento Regionale Meio Ambiente - Centro Universitário de Araraquara - Uniara. 
instáveis ou radioativos. Eles estão em constante e lenta desintegração, liberando energia através de ondas eletromagnéticas (raios gama) ou partículas subatômicas com alta velocidade (partículas alfa, beta e nêutrons) (FARIA et al., 1999).

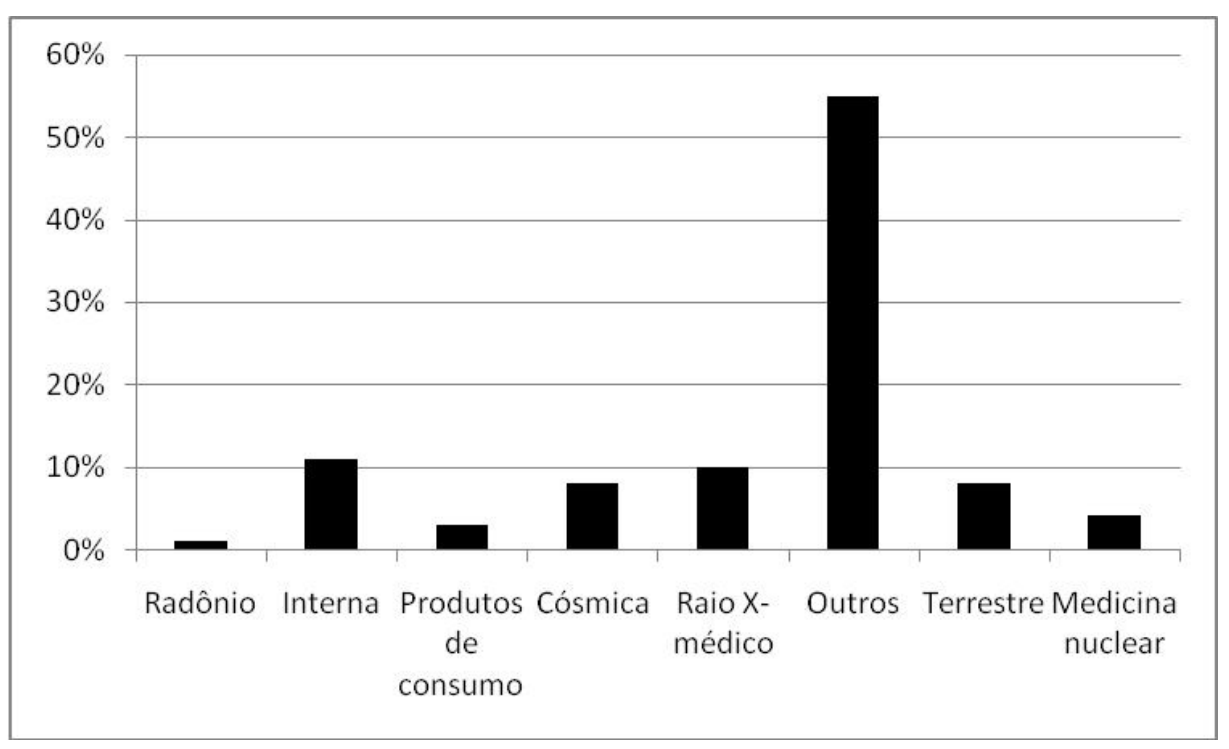

Figura 1. Principais fontes de radiação que atuam sobre o homem (FARIA et al., 1999).

Os marcos da história da radioatividade foram recentemente descritos na literatura, salientando a importância da radiação e da energia nuclear como uma ferramenta extremamente útil para o enfrentamento de problemas da humanidade, sendo a medicina nuclear a área que vem demonstrando maior utilização da energia nuclear. Entretanto, o uso de materiais radioativos em um grande número de atividades vem exigindo investigações científicas para solucionar as dificuldades técnicas de sua aplicação e assegurar sua utilização de forma segura em benefício dos seres vivos e evitando impactos ambientais. (XAVIER et al., 2007).

Os efeitos da radioatividade no ser humano dependem da quantidade acumulada no organismo e do tipo de radiação. A radioatividade é inofensiva em pequenas doses, porém, pode provocar sérias lesões e, em alguns casos, resultar em óbito. É importante ressaltar que os problemas de saúde decorrentes dos efeitos da radioatividade poderão ser apresentados pelos descendentes do indivíduo que sofreu alguma alteração genética induzida pela radioatividade (CARDOSO, 2005). Nesse sentido, o objetivo da proteção radiológica é proteger os indivíduos e seus descendentes dos efeitos danosos das radiações ionizantes, permitindo a realização das atividades que fazem uso das radiações (MAZZILI, 2002).

O principal marco da proteção radiológica foi a formação da Comissão Internacional de proteção Radiológica (Internacional Comission Radiological Protection - ICRP), que elaborou recomendações sobre a utilização segura de materiais radioativos e sugeriu os limites de dose (CIPRIANI, 2002).

Posteriormente, outros grupos foram criados com o objetivo de aprofundar os estudos nessa área, entre os quais se destacam o Comitê Científico das Nações Unidas Sobre os Efeitos da Radiação Atômica (United Nations Scientific Committee on the Effects of Atomic Radiation - UNSCEAR - 1955) e a Internacional Atomic Energy Agency (IAEA - 1957). Este último é o órgão oficial da ONU, que promove a utilização pacífica de energia nuclear pelos países-membros e tem publicado padrões de segurança e normas para manuseio de materiais radioativos, transporte e monitoração ambiental.

No Brasil, a utilização das radiações ionizantes e dos materiais radioativos e nucleares é regulamentada pela Comissão Nacional de Energia Nuclear (CNEN), autarquia federal criada em 10 de outubro de 1956 e vinculada ao Ministério de Ciência e Tecnologia, a quem compete a descrição das normas de controle que cobrem as atividades relativas ao gerenciamento de material radioativo, da origem ao destino final (RISSATO, 2007).

Todas as atividades que utilizam material radioativo devem ser submetidas a um processo de licenciamento que obedece a três princípios básicos de radioproteção: justificação (uso do material radioativo deve trazer benefícios para a sociedade) otimização (exposição à radiação ionizante, ocupacional ou pública, deve ser mantida em valor tão baixo quanto razoavelmente exeqüível, levando em conta aspectos econômicos e sociais); limitação de dose (as exposições devem obedecer aos limites de dose definidos por lei. A Tabela 1 apresenta os valores limites de dose de radiação, estabelecidos por legislação nacional (CNEN-NE 3.01, 2005) e internacional (IAEA, 2002) para exposição médica, ocupacional e do público. É importante ressaltar que doses iguais ou superiores a $6.000 \mathrm{mSv}$ são fatais e doses da ordem de $4.000 \mathrm{mSv}$ provocam doenças temporárias (CIPRIANI, 2002). 
Tabela 1. Comparação de valores de doses e seus efeitos com os limites de doses internacionalmente estabelecidos.

\begin{tabular}{|l|l|l|}
\hline Efeitos & Valores de dose \\
\hline \multirow{2}{*}{$\begin{array}{l}\text { Limite de dose para o } \\
\text { trabalhador }\end{array}$} & Média em 5 anos & $20 \mathrm{mSv} / \mathrm{ano}$ \\
\cline { 2 - 3 } & Em um ano & $50 \mathrm{mSv}$ \\
\hline Dose típica de trabalhadores & $3 \mathrm{mSv} / \mathrm{ano}$ \\
\hline Dose de tripulação aérea & De 3 a $4 \mathrm{mSv} / \mathrm{ano}$ \\
\hline \multirow{2}{*}{ Limite de dose para o público } & Média & $1 \mathrm{mSv} / \mathrm{ano}$ \\
\cline { 2 - 3 } & Num único evento & $5 \mathrm{mSv} / \mathrm{ano}$ \\
\hline \multirow{2}{*}{ Radiação de fundo da terra } & Normal & De 1 a $2 \mathrm{mSv} / \mathrm{ano}$ \\
\cline { 2 - 3 } & Em algumas regiões & De 10 a $20 \mathrm{mSv} / \mathrm{ano}$ \\
\hline Dose de raio-x em exames médicos & Até $20 \mathrm{mSv} / \mathrm{ano}$ \\
\hline \multirow{2}{*}{$\begin{array}{l}\text { Liberação de radioatividade } \\
\text { por reatores nucleares }\end{array}$} & Limite autorizado & $0,120 \mathrm{mSv} / \mathrm{ano}$ \\
\cline { 2 - 3 } & Liberações normais & $0,00220 \mathrm{mSv} / \mathrm{ano}$ \\
\hline \multicolumn{2}{|l|}{ Dose média global devida a todas as fontes } & $5.20 \mathrm{mSv} / \mathrm{ano}$ \\
\hline
\end{tabular}

Fonte: Adaptada de Cipriani (2002).

O reconhecimento dos riscos em potencial, na manipulação dos elementos radioativos, põe em evidência a necessidade de se determinar o nível de radiação nas atividades que requerem uso de materiais radioativos por meio de procedimentos de monitoração, como por exemplo, na iodoterapia.

\section{Iodoterapia}

A iodoterapia é a terapêutica que utiliza os isótopos radioativos do 123I e 131I para tratamento em cintilografia da tireóide, rastreamento de metástases e origem tireoideana, terapêutica de hipertireoidismo, doses ablativas e terapêuticas para tratamento do câncer diferenciado da tireóide (CTD) (OLIVEIRA et al., 1987; CALEGARO, 2007). Desde 1950, o radioisótopo 131I é um dos radionuclídeos mais utilizados na medicina nuclear, considerado "traçador universal", e o primeiro radionuclídeo aprovado para uso nos Estados Unidos pelo Conselho Nacional de Monitoramento e Proteção Radioativa (Nacional Council on Radiation Protection and Measurements-NCPR 1982), na forma de iodeto de sódio.

$\mathrm{O}$ valor do tratamento combinado para CTD, cirurgia seguida de tratamento com 131I e hormônios tireoidianos, é reconhecido desde 1980, pela observação da intensa diminuição do índice de recidivas, após a utilização dessa terapêutica, que envolve a iodoterapia (CALEGARO, 2007).
Essa terapêutica pode ser realizada tanto em ambulatório, quando o paciente recebe a dose radioativa na unidade de medicina nuclear, sendo orientado para ficar em isolamento no seu domicílio, quanto em unidade de internação hospitalar. O requisito básico que justifica a internação é a aplicação do 131I com atividade acima de 1,11 Gigabecquerel (Gbq) (30 milicurie), conforme norma estabelecida pela Comissão Nacional de Energia Nuclear (CNEN-NE-3.05./96).

As normas que regem a biossegurança do elemento 131I devem ser consideradas pelos profissionais da medicina nucleare da unidade de internação, que compõem a equipe de enfermagem (Enfermeiros, Técnicos de Enfermagem e Auxiliar de Enfermagem), profissionais atuantes diretamente na unidade de internação.

O conhecimento técnico referente aos procedimentos utilizados para o preparo do quarto de internação, antes, durante e após o tratamento com o 131I, e à adequação das instalações intra-hospitalares, principalmente quanto ao decaimento e armazenamento dos rejeitos radioativos, gerados durante o período da internação, é de fundamental importância para a proteção de pacientes e profissionais da saúde. A orientação e a utilização adequadas dos princípios de proteção radiológica, pela equipe de enfermagem na iodoterapia, fornecem proteção efetiva aos profissionais envolvidos no tratamento de pacientes com 131I (CALEGARO, 2007).

\section{Rejeitos Radioativos}

Os Resíduos de Serviço de Saúde, embora constituam uma parcela de cerca de $2 \%$ em relação ao volume total dos resíduos urbanos gerados, oferecem riscos de exposição, tanto aos trabalhadores quanto aos usuários, principalmente pelos resíduos infectantes e pelos rejeitos radioativos. Uma sistemática revisão da literatura realizada por Takayanagui et al (2005), na qual foram discutidos os riscos relacionados à periculosidade dos resíduos de serviços de saúde, contempla apenas duas investigações sobre resíduos radioativos.

Os rejeitos radioativos são gerados pelos estabelecimentos prestadores de serviços de saúde, resultantes do uso de substâncias radioativas não-seladas para fins terapêuticos, de diagnóstico e de pesquisa (CUSSIOL, 1999). Essa geração se deve tanto à realização de trabalhos planejados como às tarefas de limpeza de materiais e de áreas de trabalho em situações de incidente, tais como derramamentos e vômitos de pacientes tratados com radioisótopo.

Investigações sobre os procedimentos que envolvem o descarte do lixo radioativo gerado na iodoterapia são muito pouco discutidas, como apontado por Mateus (2000) e Thompson (2001). Esses autores alertam para a importância das ações da equipe de enfermagem, tanto nas precauções e gerenciamento da hospitalização na iodoterapia, quanto ao processo de decaimento e destino dos rejeitos radioativos 
gerados no período de internação.

A dosagem de 131I, utilizada no tratamento das patologias oncológicas, varia de $100 \mathrm{mCi}$, nos casos apenas de tecidos remanescentes após cirurgias, até $250 \mathrm{mCi}$ ou mais, para tratamento de metástases à distância. No momento em que o paciente recebe a dose de 131I acima de $30 \mathrm{mCi}$, ele deve permanecer internado, em unidade hospitalar, com acomodações que atendam à normatização da Comissão Nacional de Energia Nuclear - CNEN-NE 6.05.

Após a ingestão do 131I, em forma líquida ou em cápsulas, a absorção pelo organismo humano ocorre rapidamente, interagindo com o metabolismo corpóreo. Apenas parte do 131I ingerido é absorvida pela tireóide ou por células metastáticas e o restante é eliminado pelas excretas orgânicas (saliva, urina, fezes, suor e vômito), 24 horas após a ingestão, perdurando por um período médio de cinco dias.

Antes, durante e após a terapêutica, o paciente e o ambiente de internação devem ser monitorados, geralmente pelo sistema contador Geiger-Muler, sob a responsabilidade do físico da medicina nuclear. A monitoração individual ou de exposição (região corpórea do paciente) dará subsídios para liberação ou alta do paciente, que poderá ser liberado quando a atividade presente de $131 \mathrm{I}$ for igual ou inferior a $1,11 \mathrm{Gbq}(30 \mathrm{mCi})$.

A monitoração do ambiente de internação deve ser realizada nos pontos de riscos da área física (locais de maior contato do paciente, protegidos com revestimento plástico: maçanetas das portas, maçanetas do armário, torneira do quarto e do banheiro, interruptor do quarto e do banheiro, ralo do box e mesa), roupas de cama (lençole fronha) e outros rejeitos (restos alimentares, lixo do quarto e do banheiro, os plásticos utilizados para a proteção dos pontos de risco, material utilizado para descontaminação do quarto após a alta). No caso de contaminação, esses materiais devem ser armazenados em local apropriado, para sofrer o processo de decaimento, até atingir níveis aceitáveis pela normatização CNEN-NE-6.05/96 D.O.U. de 17/12/85.

Os estabelecimentos geradores de rejeitos radioativos devem possuir capacidade técnica para coletar, caracterizar e segregar os rejeitos bem como armazená-los para o processo de decaimento. Além disso, devem ser capazes de realizar avaliações que garantam que os rejeitos a serem liberados estejam em conformidade com os limites de eliminação estabelecidos nas normas vigentes. A liberação dos rejeitos radioativos para o sistema de coleta urbano e para a lavanderia, no caso do enxoval da unidade hospitalar, só deve ser realizada quando a atividade de irradiação específica do 131 I atingir o nível de irradiação, valor de $2 \mathrm{mCi} / \mathrm{kg}$, após ser submetido ao processo de decaimento (CIPRIANI, 2002).

O gerenciamento de rejeitos radioativos compreende sucessivas etapas, abrangendo desde a geração até a deposição, incluindo a coleta, segregação dos

REVISTA UNIARA, $n .^{0} 21 / 22,2008 / 2009$ diversos tipos, transporte, caracterização, tratamento, armazenamento, decaimento e a disposição final (UNIFESP, 2007), e exige das unidades hospitalares a implantação e execução de um Programa de Gerenciamento de Rejeitos Radioativos (PGRR). O fluxograma de Gerenciamento de Rejeitos Radioativos de Serviços de Saúde, Intra-hospitalar (Figura 2), ilustra todas as etapas pelas quais os rejeitos radioativos gerados em unidades de saúde devem ser submetidos, antes de ser descartados, salientando-se a etapa de descontaminação pelo decaimento.

A implantação de um PGRR é de responsabilidade da direção do estabelecimento prestador de saúde e não deve contemplar apenas as etapas experimentais dos procedimentos a serem adotados, mas também definir e documentar as responsabilidades da equipe de profissionais envolvidos na gerência dos rejeitos radioativos por meio de Procedimentos Operacionais Padrão (POPs).

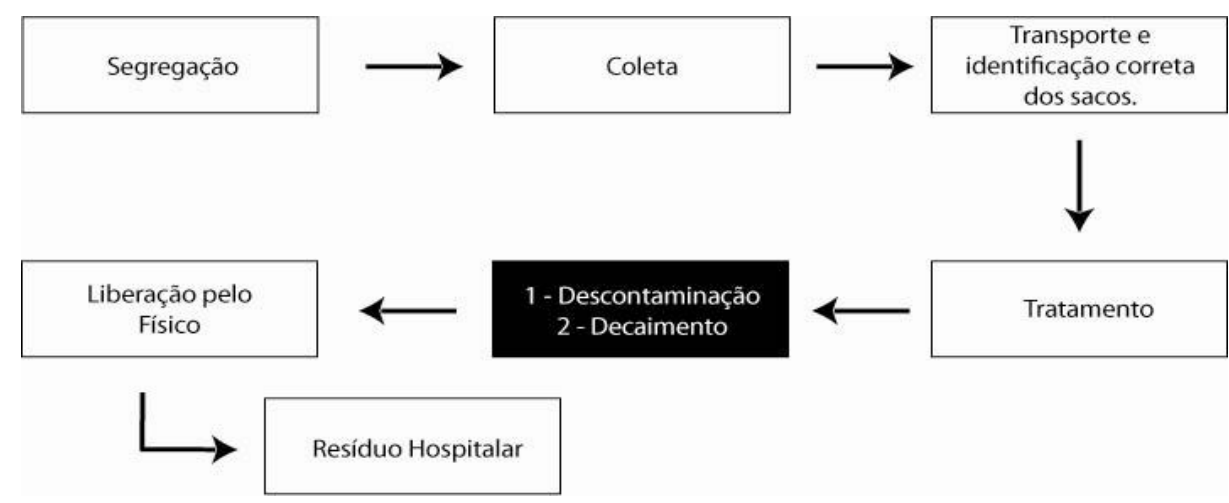

Figura 2. Gerenciamento dos Rejeitos Radioativos Intra-hospitalar. Adaptada pela autora (CUSSIOL 1999; MAZZILLI 2002; UNIFESP 2007).

Uma ferramenta de trabalho que as unidades hospitalares devem disponibilizar para a equipe de enfermagem é um Manual de Procedimentos Operacionais Padrão da Iodoterapia, que discuta conceitos básicos e procedimentos operacionais desta terapêutica, de modo a proporcionar proteção aos profissionais da saúde, aos pacientes e ao meio ambiente.

A importância do treinamento dos profissionais envolvidos nas atividades de radiação é ressaltada por Thompson (2001), que trabalhou com iodoterapia durante 20 anos, sugerindo a elaboração de manuais e vídeos como referências educacionais para que fiquem permanentemente disponíveis no posto de enfermagem na unidade de internação. A implantação de um programa de educação continuada, com embasamento técnico, é considerada uma exigência legal, como prevê a resolução SS n. 625 de 14/12/1994 no inciso 9.3.1 f, uma vez que noções básicas sobre rejeitos radioativos não estão 
incluídas na grade curricular dos profissionais de enfermagem, conforme Legislação do Ensino Superior 2001, Resolução n. ${ }^{\circ} 3$ de 07 de novembro de 2001, Diretrizes Curriculares Nacionais dos Cursos de Graduação de Enfermagem, Medicina e Nutrição. Uma proposta de roteiro, que abrange tópicos para elaboração de um manual que forneça subsídios para programas de treinamento dos profissionais de saúde envolvidos nessa atividade, está apresentado no Quadro 1.

\begin{tabular}{|c|l|}
\hline Conceitos & $\begin{array}{l}\text { Medicina nuclear; radioisótopos; iodoterapia; características do }{ }^{131} \text { I; fontes de } \\
\text { radiação; irradiação; contaminação. }\end{array}$ \\
\hline $\begin{array}{c}\text { Dose e via de } \\
\text { administração }\end{array}$ & $\begin{array}{l}\text { Dose para diagnóstico (Cintilografia); doses terapêuticas para pacientes } \\
\text { ambulatoriais e internados; via de administração; transporte da dose. }\end{array}$ \\
\hline $\begin{array}{c}\text { Legislação } \\
\text { Vigente }\end{array}$ & CNEN-NE; CONAMA e ANVISA; radioproteção; fonte. \\
\hline $\begin{array}{c}\text { Rejeitos } \\
\text { radioativos }\end{array}$ & $\begin{array}{l}\text { Definição; vias de eliminação; monitoramento; prevenção e controle da } \\
\text { contaminação radioativa; prevenção e controle à exposição radioativa; limites } \\
\text { de dose; dosímetro. }\end{array}$ \\
\hline $\begin{array}{c}\text { Procedimentos } \\
\text { na Internação }\end{array}$ & $\begin{array}{l}\text { Preparo do quarto antes da internação; paramentação; instruções ao paciente; } \\
\text { visitas; cuidados de enfermagem antes, durante e após o período de internação; } \\
\text { alta radiologica; descontaminação do quarto; cuidados em caso de acidente no } \\
\text { quarto. }\end{array}$ \\
\hline $\begin{array}{c}\text { Gerenciamento } \\
\text { dos Rejeitos } \\
\text { radioativos }\end{array}$ & $\begin{array}{l}\text { Tratamento dos rejeitos radioativos; meia vida do rejeito radioativo; depósitos } \\
\text { dos rejeitos radioativos; critérios para liberação dos rejeitos radioativos. }\end{array}$ \\
\hline
\end{tabular}

Quadro 1. Roteiro para elaboração de um manual em iodoterapia.

\section{Considerações finais}

O manuseio adequado de resíduos radioativos gerados na terapêutica da iodoterapia depende do conhecimento específico do profissional responsável pelo serviço de medicina nuclear, das ações da direção do estabelecimento prestador de saúde e da fiscalização dos órgãos competentes.

\section{Referências bibliográficas:}

CALEGARO, J.U.M.;TEIXEIRA, S.M.P.Avaliação da exposição ocupacional de auxiliares de enfermagem na iodoterapia durante 11 anos. Radiologia Brasileira v.40, p.263-266, 2007.

CARDOSO,Eliezer M.Programa de informação CNEN, módulo informação técnica. 2005. Disponívelem: <www.cnen.gov.br>.Acessoem: mar. 2009.

CIPRIANI, M. Mitigação dos impactos sociais e ambientais decorrentes do fechamento definitivo de minas de urânio. 2002. 331f. Tese (Doutorado em Administraçãoe Política de recursos Minerais), Unicamp, Campinas, 2002.

REVISTA UNIARA, $n .^{0} 21 / 22,2008 / 2009$
CONSELHONACIONAL DEENERGIA NUCLEAR. Norma de Requisitos de Radioproteção e Segurança para Serviços de Medicina Nuclear. $01 / 2005$

Norma de Diretrizes Básicas de Proteção Radiológica-NE-3.01, CNEN

Norma de Requisitos de Radioproteção e Segurança para Serviços de Medicina Nuclear-NE-3.05, CNEN, 03/1996.

. Norma de Gerência de Rejeitos Radioativos em Instalações Radioativas NE-6.05, CNEN, 10/1996.

CUSSIOL, N.A.M., Silva, E.M.P. Gerência de rejeitos radioativos de serviços de saúde. Comissão Nacional de Energia Nuclear e Centro de Desenvolvimento da Tecnologia Nuclear. Belo Horizonte, 1999. Publicação CDTN-857/99.

FARIA, Rafael; TAMARA, A.; ROCHA, F.; ANTUNES, S.

Radioatividade: conceitos fundamentais. Belo Horizonte, Universidade Federal de Minas Gerais. (Departamento de Fisiologia e Biofísica), 1999. Disponível em<http: //radio_teleterapia.vilabol.uol.com.br/radioatividade> . Acesso em: mar. 2009.

IAEA-Internacional Atomic Energy Agency. Safety, Standards,Series. Radiological Protection for Medical Exposure to Ionizing Radiation. Vienna, 2002.

MATEUS, L. Aimportância da enfermagem no tratamento com iodo radioativo. Nursing. São Paulo, n. 25, p.6-8, 2000

\section{MAZZILLI, B.P. et al. Noções básicas de proteção radiológica. IPEN, 2002.}

OLIVEIRA, E.R., ESTEVES, R.N., MARINHO, J.C.A., B.A.; CORREA, N.D., Nascimento, Manual do serviço de medicina nuclear do Hospital dos Servidores do Estado do Rio de Janeiro. Rio de Janeiro:HSE, 1987.

Resolução SS-625, de 14-12-94. Aprova norma técnica que dispõe sobre o Uso, posse e armazenamento de fontes de radiação ionizante no âmbito do Estado de São Paulo. Disponívelem: <http://fosjs.unesp.br/radiologia/legislaçãoraiox/ resolução/\%20SS-625-1-1994>. Acesso em: abr. 2006. 
RISSATO, M.L. Iodoterapia: avaliação crítica de procedimentos de precauçãoe manuseio dos rejeitos radioativos gerados em unidades de internação

hospitalar. 2007. 131f. Dissertação (Mestrado em Desenvolvimento Regional e Meio Ambiente), UNIARA, Araraquara, 2007.

TAKAYANAGUI,A.M.M.; LOPES, T.M.; SEGURA-MUÑOZ, S. O conhecimento científico sobre riscos ligados a resíduos de serviços de saúde obtido por meio de revisão sistemática de literatura. In: INTERNATIONAL SOLID WASTEASSOCIATION. (Exposicion y Congresso Mundial:Hacia um sistema integral de resíduos sólidos), BuenosAires, 2005.

THOMPSON, M.A., Radiation Safety Precautions in the Manegement of the Hospitalized 131ITherapyPatient. Journal of Nuclear Medicine Technology, Alabama, v.29, n.2, June 2001 .

UNIFESP - Universidade Federal de São Paulo Escola Paulista de Medicina - Núcleo deProteção Radiológica.Disponívelem:http:/protecaoradiologica.unifesp.br/ somos.php. Acesso em: 10 abr. 2009.

XAVIER,A.M.; LIMA,A.G; VIGNA, C.R.M.;VERBI,F.M.; BORTOLETO,GG; GORAIEB,K.; COLLINS, C.H.; BUENO, M.I.M. Marcos da história da radioatividade e tendências atuais. Química Nova. v.30., p.83-91, 2007.

Title:

IMPORTANCE OF MANAGEMENT OF RADIOACTIVE RESIDUES IN IODOTHERAPY.

\section{Abstract:}

Iodotherapy is one of the medicinal nuclear therapeutics that employs radioactive iodine as the radionuclide. This treatment, carried out in hospitals, requires radioprotection procedures to guard the patients, the medical team and the environment against contamination. A discussion regarding the application of this therapeutic shows the importance of implanting programs to manage radioactive residues which are generated in hospital units.

Keywords: Iodotherapy, Nuclear Medicine, Radioactive Residues. 